\title{
Efeitos do género, tempo de serviço e orientação religiosa da escola na dimensão ética e afetiva da identidade docente
}

\author{
Manuel Granjo ${ }^{1} \&$ Francisco Peixoto ${ }^{1}$ \\ ${ }^{1}$ Centro de Investigação em Educação - ISPA / ISPA - Instituto Universitário
}

\begin{abstract}
Resumo: Este estudo analisa as diferenças introduzidas pelo género, anos de serviço e orientação religiosa da escola na dimensão ética e afetiva da identidade docente. 450 professores de escolas privadas distribuídas pelo território nacional e ilha da Madeira, sendo 306 do género feminino (68\%), com idades entre os 21 e os 73 anos $(M=41, D P=9.56)$ e uma média de 15.5 anos de serviço, responderam a um questionário online. Os resultados mostram que as professoras expressaram autopercepções mais favoráveis na orientação ética, na relação com os colegas e satisfação, nas competências profissionais e de conduta e no compromisso afetivo. Os anos de serviço introduzem diferenças no autoconceito profissional e em algumas das suas dimensões; o compromisso afetivo aumenta com a experiência, enquanto o compromisso instrumental diminui. Os professores de escolas com orientação religiosa apresentam autopercepções mais favoráveis na relação com os alunos, na satisfação profissional e no compromisso.
\end{abstract}

\section{Palavras-chave: Identidade docente; género; tempo de serviço; orientação religiosa da escola.}

Effects of gender, teaching experience and school religious orientation on ethical and affective dimensions of teacher identity: This study analyzes the differences introduced by gender, teaching experience and school religious orientation on ethical and affective dimensions of teacher identity. An online questionnaire was filled by 450 Portuguese teachers (68\% female) aged 21 to 73 years $(M=41, S D$ =9.56). Teachers were from private schools all around the Portuguese territory and Madeira Island, and had an average of 15.5 years of teaching experience $(S D=9.47)$. The results showed that female teachers express more favourable self-perceptions in ethical orientation, professional knowledge and conduct, and in the affective commitment to the school. Teaching experience introduced differences in professional self-concept and some of its dimensions; the affective commitment increases with experience, favouring the more experienced group, while the instrumental commitment decreases. Teachers in schools with religious orientation presented more favourable self-perceptions in their relationship with students, their satisfaction, and their affective commitment to the school.

Keywords: Teacher identity; gender; teaching experience; school religious orientation.

A identidade docente e o seu desenvolvimento é um tema estruturante na reflexão sobre transformação na educação. Ela é o cerne da profissão docente, ao fornecer uma estrutura a partir da qual os professores constroem as suas ideias de "como ser", "como agir", e "como entender" o seu trabalho e o seu lugar na sociedade (Sachs, 2005, p.15). Tratando-se de um construto polissémico, é possível encontrar na literatura existente outros conceitos que se inter-relacionam e nos ajudam a melhor compreendê-la, designadamente as suas relações com a ética, o autoconceito profissional, a autoestima, a motivação e o compromisso do professor (e.g., Beijaard, Meijer, \& Verloop, 2004; Canrinus, Helms-Lorenz, Beijaard, Buitink, \& Hofman, 2012; Kelchtermans, 2005). Neste âmbito, a orientação ética é definida como um conjunto de princípios que guiam o professor no sentido de discriminar o certo do errado, o bem do mal e o moral do imoral, ajudando-o na escolha de um modo adequado de conduta (e.g., Korthagen, 2004; Sochett, 2008). A maturidade ética do professor pode caracterizar-se pela habilidade em manter a perspetiva do cuidado e da justiça de forma equilibrada e complementar (e.g., Banks \& Nohr, 2003; Benhabit, 1992; Jaffe \& Hyde, 2000; Noddings, 1999; Rawls, 1971/1993). Por sua vez, o autoconceito profissional dos professores remete para um conjunto de crenças sobre aspectos relativos à autorealização no processo ensino-aprendizagem, na relação com os alunos e professores e ao valor reforçador da profissão docente (e.g., Gonçalves \& Veiga, 2006; Markus \& Wurf, 1987; Marsh \& Ayotte, 2003; Villa \& Calvete, 2001). A competência percebida do professor representa a perceção que o professor

${ }^{1}$ Morada para correspondência: Francisco Peixoto, ISPA - Instituto Universitário, R. Jardim do Tabaco, 34, 1149 - 041 Lisboa, Portugal. Email: manuelgranjo@hotmail.com 
possui das suas práticas, a partir da autoperceção das suas competências profissionais (e.g., Bong \& Skaalvik, 2003; Marsh \& Roche, 2000). A autoestima é definida como uma autoperceção global do valor do indivíduo - independente de domínios de competência -, de satisfação consigo próprio (e.g., Messer \& Harter, 1986). 0 construto satisfação de necessidades psicológicas básicas no trabalho do professor assenta no pressuposto da teoria da autodeterminação de que a satisfação de três necessidades básicas inatas e universais - autonomia, competência e relacionamento - contribui para a expressão das capacidades e competências pessoais e para a vivência de experiências de autodeterminação (Deci \& Ryan, 2000). Por fim, o compromisso com a escola ${ }^{2}$ remete para a identificação do professor com a missão e os valores da escola, a que se alia um elevado sentido de comunidade e o desejo de nela permanecer, manifesto em três dimensões diferentes: o compromisso afetivo, o compromisso normativo e o compromisso instrumental (e.g., Myer \& Allen, 1991; Razak, Darmawan, \& Keeves, 2009). Já o compromisso com a profissão reenvia para a identificação e satisfação do professor com a docência, uma força que o incita a melhorar as suas competências profissionais, independentemente do estabelecimento de ensino, e a não abandonar a profissão (e.g., Skaalvik \& Skaalvik, 2011).

A literatura sobre a dimensão ética e afetiva da identidade docente, para além de escassa, no que à dimensão ética diz respeito, revela resultados não totalmente concordantes quanto aos efeitos de variáveis individuais e de contexto.

De seguida, apresentamos os resultados da revisão de literatura efetuada, sendo que neste estudo pretendemos aprofundá-los, por forma a melhor entender essas relações e, por outro lado, deles retirar ilações teóricas e práticas para o desenvolvimento da identidade docente e as práticas dos professores.

\section{Efeitos do género sobre variáveis associadas à identidade docente}

No âmbito da orientação ética, Gilligan (1982) defende a existência de dois tipos de orientações éticas distintas, em que os homens tomariam as suas decisões orientadas por princípios baseados na ética da justiça, enquanto as mulheres deliberariam com base em princípios da ética do cuidado. Apesar de homens e mulheres tomarem decisões com base nos princípios da ética do cuidado e da ética da justiça, alguns autores defendem que as deliberações orientadas para o cuidado são utilizadas predominantemente pelas mulheres (e.g., Gilligan, 1982; Gilligan \& Attanucci, 1988; Jaffe \& Hyde, 2000; Karniol, Grosz, \& Schorr, 2003). Ao invés, Kohlberg (1984) sustenta que a ética do cuidado e a ética da justiça não representam formas distintas de desenvolvimento moral. Jaffe e Hyde (2000), a partir de uma exaustiva revisão de literatura relativa à polémica Gilligan vs. Kohlberg, salientam que apesar de haver alguma predominância da ética do cuidado nas mulheres e da ética da justiça nos homens, o que caracteriza verdadeiramente a maturidade ética de uma pessoa é a habilidade de manter a perspetiva do cuidado e da justiça de forma equilibrada e complementar, o que está em consonância com o pensamento de Benhabit (1992) e Noddings (1999). Acresce, ainda, a possibilidade de encontrar autores que invocam a inexistência de diferenças significativas entre os géneros na orientação ética (e.g., Vikan, Camino, \& Biaggio, 2005).

Relativamente ao autoconceito profissional dos professores, a maioria dos estudos revelam que os professores do género masculino apresentam níveis mais elevados no autoconceito profissional e em algumas das suas dimensões do que os professores do género feminino (Fonseca \& Veiga, 2010; Gonçalves \& Veiga, 2006; Marsh \& Roche, 2000; Veiga, Roque, Antunes, Fernandes, \& Guerra, 2003; Villa \& Calvete, 2001). Igualmente, estudos sobre a autoeficácia docente encontram resultados que vão no mesmo sentido (Antoniou, Polychroni, \& Vlachakis, 2006; Klasser \& Chiu, 2010; Wolters \& Daugherty, 2007). No que concerne aos efeitos de género sobre a autoestima, a maioria dos estudos realizados referem a inexistência de diferenças nas autorepresentações globais introduzidas pelo género (e.g., Hattie, 1992; Peixoto, 2003; Wylie, 1979). No entanto, outros estudos referem que as diferenças favorecem os indivíduos do género masculino (e.g., Aznar, Fernández, Quevedo, \& Abella, 2003; Harter, 1999). De forma mais reduzida, é possível ainda encontrar estudos em que os efeitos do género favorecem os indivíduos do género feminino (e.g., Ruivo, Sebastião, Rafael, Afonso, \& Nunes, 2008; Tabassum \& Ali, 2012).

Em relação aos efeitos do género no compromisso, há autores que apresentam resultados em que os indivíduos do género masculino apresentam níveis de compromisso organizacional significativamente mais elevados, justificados por usufruírem de melhores remunerações salariais e ocuparem melhores posições ocupacionais (e.g., Aydin, Sarier, \& Uysal, 2011). Por sua vez, outros autores referem nos seus

\footnotetext{
2 Optámos por utilizar a tradução compromisso da palavra inglesa «Commitment», ao invés da palavra comprometimento utilizada por outros autores (e.g., Nascimento, Lopes \& Salgueiro, 2008; Rego, Cunha \& Souto, 2007). Embora ambas as palavras sejam sinónimas, e não havendo sinónimos perfeitos na língua portuguesa, parece-nos que a palavra compromisso expressa melhor o vínculo do professor com a organização escolar como um todo, expressando a obrigação, o desejo e a necessidade do professor permanecer na escola, em consonância com o "Modelo das Três Componentes" de Meyer e Allen (1991).
} 
estudos que as mulheres apresentam um compromisso significativamente superior em relação aos homens, que atribuem ao facto de as mulheres terem tido que lutar arduamente para conseguirem um estatuto semelhante ao dos homens (e.g., Mowday, Steers, \& Porter, 1979). Por sua vez, Balay (2000) apresenta resultados em que o género não tem qualquer efeito sobre o compromisso.

\section{Efeitos do tempo de serviço sobre variáveis associadas à identidade docente}

No que se refere aos efeitos dos anos de serviço/experiência sobre o autoconceito profissional, os resultados obtidos por Veiga et al. (2003) evidenciaram que o autoconceito dos professores é mais elevado nos docentes com mais tempo de serviço, nas dimensões relação com os alunos, satisfação, aceitação de riscos e iniciativas e no autoconceito global. Ao invés, os resultados do estudo de Fonseca e Veiga (2010) revelam que o autoconceito profissional dos professores se torna mais «negativo» à medida que aumenta o tempo de serviço. Por sua vez, Gonçalves e Veiga (2006) não observaram diferenças significativas no contraste de tempo de serviço entre os grupos de professores com menos de 9 anos, entre 9 e 15 anos e com mais de 15 anos. Deste modo, podemos constatar a existência de resultados contraditórios nos resultados na investigação com professores portugueses.

No âmbito da autoeficácia, os investigadores manifestam também uma certa discordância quanto aos efeitos da experiência na competência profissional percebida. Essa discordância parece estar relacionada com a forma como a variável anos de serviço e/ou experiência docente é operacionalizada pelos diversos autores, para além da pouca evidência existente sobre o modo como as crenças de autoeficácia dos professores se modificam ou solidificam ao longo das fases da carreira profissional (Tschannen-Moran, Hoy, \& Hoy, 1998). Autores de referência, como Tschannen-Moran e Hoy (2007) ou Wolters e Daugherty (2007), sustentam que na fase inicial da carreira a autoeficácia dos professores é mais frágil e maleável, aumentando e tornando-se mais firme à medida que os professores ganham experiência profissional. Por seu turno, Kooij, de Lange, Jansen e Dikkers (2008) sustentam que os desafios com os quais se confrontam os professores nas fases da carreira média e tardia podem influenciar a motivação e a satisfação no trabalho.

Os resultados dos estudos relativos aos efeitos da experiência na motivação intrínseca dos professores revelam-se inconclusivos. Por um lado, os trabalhos de Wolters e Daugherty (2007) e Canrinus et al. (2012) referem que a experiência profissional não introduz mudanças nos níveis motivacionais dos professores (autonomia, competência/autoeficácia, relacionamento e satisfação no trabalho). Por outro, os estudos de Klasser e Chiu (2010), Day e Gu (2007) sugerem que a meio da carreira (8-23 anos) os níveis motivacionais aumentam e a partir dos 24 anos (ou mais) de experiência profissional tendem a diminuir, em linha com os estudos de Huberman (2000) e Pedro e Peixoto (2006), nos quais os professores em meio de carreira (7 a 15 anos de ensino) apresentavam níveis superiores de satisfação profissional. De forma similar, outras investigações no âmbito da satisfação profissional dos professores mostram que esta tende a ser mais elevada nos professores mais experientes (Gonçalves, 2000; Ruivo et al., 2008). Há, no entanto, autores que defendem o seu contrário, sustentando que a satisfação dos professores diminui com os anos de experiência, com os professores mais experientes a manifestarem menor satisfação no trabalho (Gursel, Sunbul, \& Sari, 2002). Por sua vez, é possível ainda encontrar um terceiro posicionamento, cujos autores defendem serem os professores em meio de carreira os que apresentam maior desmotivação e tensões profissionais (e.g., Chaplain, 1995).

$\mathrm{Na}$ literatura existente relativa aos efeitos da idade e do tempo de serviço sobre a autoestima e/ou outras representações globais sobre si próprio com participantes de diferentes faixas etárias, podemos constatar também uma certa dissonância nos resultados obtidos pelos diversos investigadores. Por um lado, Wylie (1979) e Veiga (1995), nas revisões de literatura efetuadas não encontraram diferenças introduzidas pela idade. Por outro, é referido noutros estudos que a autoestima e outras representações globais de si próprio aumentam com a idade e/ou o tempo de serviço (e.g., Fonseca \& Veiga, 2010; Gonçalves \& Veiga, 2006; Ruivo et al., 2008). Em menor número, há autores que sustentam a diminuição do autoconceito profissional dos professores (enquanto representação global de si próprio, como profissional) à medida que aumenta o tempo de serviço (Veiga et al., 2003).

Os poucos estudos existentes sobre o efeito da experiência no compromisso dos professores, apontam para a inexistência de efeitos da experiência docente sobre o compromisso com escola (Canrinus et al., 2012; Wolters \& Daugherty, 2007) e com a profissão (Canrinus et al., 2012; Skaalvik \& Skaalvik, 2011).

\section{Contexto e identidade docente}

As perspetivas psicológicas e psicossociológicas enfatizam os efeitos externos e ambientais no desenvolvimento da identidade. Alegam que o contexto não é somente um quadro onde a interação decorre, é também uma matriz que fornece à relação um código, representações, normas de desempenho 
e rituais que permitem a relação e lhe dão significado (e.g., Bandura, 1977; Brofenbrenner, 1996; Dubar, 1997; Erikson, 1982). Daí que a identidade de um professor seja moldada na interação com os outros, no contexto profissional e na qual os valores organizacionais assumem particular relevância (e.g., Flores \& Day, 2006). A missão das escolas com orientação religiosa aponta deliberadamente para a evangelização de crianças e jovens através da educação. De forma explícita, é pedido aos professores que desempenhem as suas funções de responsabilidade profissional, mas também de animação pastoral e que se envolvam em projetos que alimentem a sua fé e o seu compromisso social. 0 que, por sua vez, responsabiliza as lideranças na formação cristã e no acompanhamento espiritual dos professores nas escolas. Ademais, no centro do currículo das escolas católicas está um programa de orientação religiosa abrangente e de acordo com as orientações da igreja. Há, portanto, uma intenção deliberada de fazer destas escolas centros de evangelização, fator que diferencia a missão das escolas com e sem orientação religiosa (e.g., Moog, 2012; Papa Francisco, 2014; Verhach, 2014).

Dito isto, faz também algum sentido a intenção de procurar identificar diferenças na dimensão ética e afetiva da identidade dos professores que exercem a docência em escolas com e sem orientação religiosa.

Assim, a partir da revisão da literatura efetuada, delineámos este estudo correlacional, com uma acentuada componente exploratória, com o propósito de responder à seguinte questão geral de investigação: Será que o género, anos de serviço e orientação religiosa da escola introduzem diferenças nas perceções expressas pelos professores:

na orientação ética?

no autoconceito profissional e na autoestima?

na competência profissional percebida?

na satisfação de necessidades básicas (autonomia, competência e relacionamento)?

e no compromisso com a escola e a profissão?

\section{MÉTODO}

\section{Participantes}

Participaram no estudo 450 professores ( $32 \%$ do sexo masculino), com uma média de idades de 41 anos $(D P=9.56)$ e 15.5 anos de serviço $(D P=9.47)$, distribuídos pelo território Nacional e ilha da Madeira (região Norte - 38.7\%, Centro - 10.5\%, Lisboa e Vale do Tejo - 44.9\%, Alentejo e Algarve - $2.7 \%$, e Madeira - 3.3\%), a lecionarem no ensino básico e secundário em escolas do ensino particular e cooperativo.

Com a intenção de explorar o papel da experiência/anos de serviço para o desenvolvimento da identidade docente, os professores participantes foram divididos em três grupos, a partir dos seus níveis de experiência: 71 professores em início de carreira (1 a 5 anos de serviço), 16\%; 158 professores experientes (6 a 14 anos de serviço), 35\%; e 221 professores muito experientes (16 ou mais anos de serviço), 49\%. Os professores foram também divididos em professores oriundos de escolas com orientação religiosa, 290 (64.4\%), e sem orientação religiosa, 160 (35.6\%), no sentido de observar as variações ocorridas pela alteração do contexto escolar.

\section{Instrumentos}

Para avaliar os construtos em estudo foi aplicado um questionário, que incluía sete medidas diferentes. A escala de Orientação ética do professor $(\alpha=.87)$, por nós construída, constituída por vinte itens, distribuídos por duas dimensões - Orientação para o cuidar, $\alpha=.82$, (10 itens, e.g.,"É nossa obrigação compreender com facilidade o ponto de vista dos outros.") e Orientação para a justiça, $\alpha=.82$, (10 itens, e.g., "Devemos preocupar-nos em explicar às pessoas as decisões que lhes dizem respeito ou as afetam."). A escala de Autoconceito profissional (Granjo, 2015), $(\alpha=.88)$, adaptada da versão original da Teacher Self-concept Evaluation Scale apresentada por Villa e Calvete (2001) e da versão adaptada da mesma escala para os professores por Veiga, Gonçalves, Caldeira, \& Roque (2006), constituída por 24 itens, distribuídos por cinco dimensões - Competência $(\alpha=.89)$ (4 itens, e.g., "Sinto-me muito competente na minha profissão."); Relação com os alunos $(\alpha=.73)$ (4 itens, e.g., "Sinto segurança na forma como me relaciono com os meus alunos."); Relação com os colegas ( $\alpha=.71$ ) (4 itens, e.g., "Gosto das relações que estabeleço com os outros no meu trabalho."); Satisfação $(\alpha=.77)$ (4 itens, e.g., "O meu trabalho como professor não me dá satisfação.") e Aceitação de riscos e iniciativas $(\alpha=.58)$ (5 itens, e.g., "As mudanças não me perturbam.").

A escala de Competência percebida (Granjo, 2015), sustentada teoricamente pelo modelo das dez competências de Perrenoud (2000), $(\alpha=.82)$, é constituída por 10 itens e três dimensões: Desenvolvimento do ensino e aprendizagem $(\alpha=.79) \quad$ (5 itens, e.g., "Na minha prática profissional sou capaz de ... utilizar mecanismos de diferenciação pedagógica de acordo com o ritmo de aprendizagem dos 
alunos."); Competências profissionais e de conduta $(\alpha=.68)$ (3 itens, e.g., "Na minha prática profissional sou capaz de...fazer respeitar as regras e o código de conduta aos alunos (manter a disciplina)."; e Participação na escola e relação com a comunidade educativa $(\alpha=.57)$ (2 itens, e.g., "Na minha prática profissional sou capaz de ... informar e envolver os pais na escola."). A escala de Satisfação de necessidades básicas no trabalho do professor (Granjo, 2015), adaptada da escala "Basic Need Satisfaction at Work" (BNSW), de Ilardi, Leone, Kasser, \& Ryan (1993), inclui 8 itens, distribuídos por duas dimensões, autonomia ( $\alpha=.78),(4$ itens, e.g., "No trabalho, tenho liberdade para expressar as minhas ideias e opiniões.") e relacionamento $(\alpha=.84)$, (4 itens, e.g., "Tenho bom relacionamento com os meus/minhas colegas de trabalho."). A escala de Autoestima ( $\alpha=.74)$, adaptada por Granjo (2015) de Messer e Harter (1986), com 5 itens (e.g., "Algumas pessoas gostam do tipo de pessoa que são."). A escala de Compromisso com a escola (Granjo, 2015), $(\alpha=.78)$, baseia-se em duas escalas de compromisso organizacional adaptadas para o contexto português, por Rego, Cunha e Souto (2007) e Nascimento, Lopes e Salgueiro (2008), a partir da escala Affective, Normative and Continuance Commitment (Allen \& Meyer, 1996). É constituída por 11 itens, distribuídos por três dimensões: compromisso afectivo ( $\alpha=.84)$, (3 itens, e.g., "Ficaria muito feliz em passar o resto da minha carreira nesta escola."); compromisso normativo ( $\alpha=.82)$, (3 itens, e.g., "Neste momento, não deixaria a minha escola porque sinto obrigações para com as pessoas que aqui trabalham."); e compromisso instrumental $(\alpha=.69)$, (5 itens, e.g., "Muito da minha vida seria afetada se decidisse sair desta escola neste momento."). Finalmente, a escala de Compromisso com a profissão (Granjo, 2015), ( $\alpha=.85)$, integra 3 itens, adaptados da escala Motivation to leave the teaching profession, de Skaalvik e Skaalvik (2011), e.g., "Se eu pudesse escolher de novo uma profissão, eu não escolheria ser professor.".

Nos estudos de validação efetuados (Granjo, 2015), todas as escalas revelaram índices de ajustamento adequados nas análises factoriais confirmatórias realizadas $\left(\chi^{2} / \mathrm{df}<4.1, \mathrm{GFI}, \mathrm{CFI}>0.9\right.$; PGFI, PCFI $>0.6$, RMSEA<0.08).

\section{Procedimento}

Os professores responderam ao questionário alojado num link criado para efeito, disponível on-line de dezembro a março de 2013. Recebemos 910 questionários, dos quais foram rejeitados 460 por não estarem preenchidos na totalidade (taxa de rejeição de 50.5\%).

\section{Análise dos dados}

A análise das respostas dos professores foi efetuada com o recurso a análises de variância multivariada (MANOVA) e univariada (ANOVA), cumprindo os requisitos de homogeneidade da variância. Foi considerado o nível de significância de .01 no teste de Levene para a homogeneidade da variância nas análises univariadas e de .001 para o teste Box M, nas análises multivariadas (Tabachnick \& Fidell, 2012). Para a MANOVA apresentaremos o resultado no critério Traço de Pillai, já que se tem revelado mais robusto que os outros indicadores (Tabachnick \& Fidell, 2012). Esporadicamente, quando a potência do teste o justificava, adotámos uma postura menos conservadora recorrendo ao indicador Maior Raiz de Roy (Marôco, 2011). Quando a MANOVA identificou efeitos significativos, procedeu-se à ANOVA para cada uma das variáveis dependentes, seguida do teste post-hoc HSD de Tukey, quando a variável independente podia assumir mais do que dois valores. Os efeitos cujo nível de significância ( $p$-value) foi inferior ou igual a .05 foram considerados estatisticamente significativos. Para analisar a dimensão do efeito foi utilizado o Eta ${ }^{2}$ parcial $\left(\eta_{\mathrm{p}}{ }^{2}\right)$, de acordo com a seguinte classificação (Marôco, 2011, a partir de Cohen, 1973, 1988): pequeno $\leq .05$; médio] .05; .25]; elevado] .25; .50]; muito elevado > .5. Por último, foi também considerado a potência do teste $(\pi)$ para detetar a adequação dos efeitos significativos, cujo valor da potência $\geq .80$ é considerado como desejado, sendo o mais típico na maioria das revistas das ciências sociais e humanas, considerar o valor $\geq .60$ (Cohen, 1992; Marôco, 2011). As análises foram realizadas utilizando o software SPSS Statistics (versão 21).

\section{RESULTADOS}

A análise de variância multivariada para a orientação ética e as suas duas dimensões (orientação para o cuidado e orientação para a justiça) revelou a inexistência de efeitos significativos introduzidos pelo género, anos de serviço e orientação religiosa da escola. Por sua vez, também a interação entre estas variáveis não teve um efeito estatisticamente significativo sobre a orientação ética e as suas duas dimensões, $V=.002, F(4,876)=.286, p=.898$. As análises univariadas, no entanto, revelaram que o género introduz um efeito marginal estatisticamente significativo sobre a orientação ética, $F(1,438)=$ 3.756, $p=.053, \eta_{p}{ }^{2}=.009, \pi=.490$, sendo a dimensão do efeito pequena. Este efeito traduz-se num valor ligeiramente mais elevado na orientação ética por parte das professoras comparativamente aos professores (Tabela 1). 
Tabela 1. Médias e desvios-padrão nas dimensões da orientação ética e na escala total em função do género, anos de serviço e orientação religiosa da escola

\begin{tabular}{|c|c|c|c|c|c|c|c|}
\hline & \multicolumn{2}{|c|}{ Género } & \multicolumn{2}{|c|}{ Orientação Religiosa } & \multicolumn{3}{|c|}{ Anos de Serviço } \\
\hline & Masculino & Feminino & Não & Sim & $1-5$ & $6-14$ & $\geq 15$ \\
\hline Cuidado & $\begin{array}{c}4.67 \\
(.610)\end{array}$ & $\begin{array}{c}4.76 \\
(.602)\end{array}$ & $\begin{array}{c}4.70 \\
(.547)\end{array}$ & $\begin{array}{c}4.75 \\
(.663)\end{array}$ & $\begin{array}{c}4.68 \\
(.525)\end{array}$ & $\begin{array}{c}4.69 \\
(.601)\end{array}$ & $\begin{array}{c}4.78 \\
(.679)\end{array}$ \\
\hline Justiça & $\begin{array}{c}5.48 \\
(.511)\end{array}$ & $\begin{array}{c}5.60 \\
(.405)\end{array}$ & $\begin{array}{c}5.58 \\
(.350)\end{array}$ & $\begin{array}{c}5.55 \\
(.488)\end{array}$ & $\begin{array}{c}5.62 \\
(.320)\end{array}$ & $\begin{array}{c}5.53 \\
(.420)\end{array}$ & $\begin{array}{c}5.56 \\
(.492)\end{array}$ \\
\hline Orientação ética & $\begin{array}{c}5.08 \\
(.509) \\
\end{array}$ & $\begin{array}{l}5.19^{*} \\
(.475)\end{array}$ & $\begin{array}{r}5.14 \\
(.385) \\
\end{array}$ & $\begin{array}{c}5.15 \\
(.511) \\
\end{array}$ & $\begin{array}{r}5.15 \\
(.369) \\
\end{array}$ & $\begin{array}{c}5.11 \\
(.443) \\
\end{array}$ & $\begin{array}{r}5.17 \\
(.514) \\
\end{array}$ \\
\hline
\end{tabular}

Nota: Entre parênteses apresenta-se o valor do desvio-padrão correspondente.

* Diferença significativa para $p<.05$

Para identificar os efeitos das variáveis independentes (género, orientação religiosa da escola e anos de serviço) relativamente ao autoconceito profissional docente e às suas seis dimensões foi igualmente realizada uma análise de variância multivariada. Os resultados revelaram que o género teve um efeito de dimensão reduzida sobre o compósito multivariado do autoconceito profissional, $V=.043$, $F(7,432)=2.739, p=.009,, \eta_{p}^{2}=.043, \pi=.910$. Recorrendo ao indicador Maior Raiz de Roy, cujo valor da potência do teste está acima do aceitável, é possível observar um efeito de interação entre a orientação religiosa e os anos de serviço sobre as dimensões do autoconceito profissional, $\lambda_{1}=.038, F(7,433)=2.377$, $p=.022, \eta_{p}^{2}=.037, \pi=.858$. Observada a significância multivariada, realizaram-se análises de variância univariada (ANOVAs) para cada uma das variáveis dependentes, seguidas do teste post-hoc de Tukey. A análise das probabilidades de significância revela que o género introduz um efeito de pequena dimensão, sobre as variáveis dependentes relação com os colegas, $F(1,438)=5.763, p=.017, \eta_{p}^{2}=.013, \pi=.668$; um efeito de média dimensão na aceitação de riscos e iniciativas, $F(1,138)=3.873, p=.050, \eta_{p}^{2}=.09, \pi=.502$, e um efeito de pequena dimensão, tendencialmente significativo, na variável satisfação, $F(1,438)=3.572$, $p=.059, \eta_{p}^{2}=.008, \pi=.502$. A análise da Tabela 2 permite constatar que as professoras apresentam uma perceção de competência mais elevada, na dimensão relação com os colegas e na dimensão satisfação, do que os professores. Ao invés, os professores percecionam-se como aceitando mais riscos e iniciativas do que as professoras.

As análises univariadas realizadas à variável independente orientação religiosa da escola mostram um efeito de pequena dimensão, estatisticamente significativo, na dimensão do autoconceito profissional docente relação com os alunos, $F(1,438)=3.894, p=.049, \eta_{p}^{2}=.009, \pi=.504$, e na dimensão satisfação, $F(1,438)=7.137, p=.008, \eta_{p}^{2}=.016, \pi=.760$. A análise da Tabela 2 permite verificar que os professores que exercem a docência em escolas com orientação religiosa apresentam resultados mais elevados nas dimensões relação com os alunos e satisfação, do que os professores que exercem a docência em escolas sem orientação religiosa.

Tabela 2. Médias e desvios-padrão nas dimensões e na escala total de autoconceito profissional docente em função do género, anos de serviço e orientação religiosa da escola

\begin{tabular}{|c|c|c|c|c|c|c|c|}
\hline & \multicolumn{2}{|c|}{ Género } & \multicolumn{2}{|c|}{ Orientação Religiosa } & \multicolumn{3}{|c|}{ Anos de Serviço } \\
\hline & Masculino & Feminino & Não & Sim & $1-5$ & $6-14$ & $\geq 15$ \\
\hline Competência & $\begin{array}{c}5.21 \\
(.576)\end{array}$ & $\begin{array}{c}5.17 \\
(.559)\end{array}$ & $\begin{array}{c}5.19 \\
(.535)\end{array}$ & $\begin{array}{c}5.18 \\
(.580)\end{array}$ & $\begin{array}{c}5.13 \\
(.570)\end{array}$ & $\begin{array}{c}5.23 \\
(.497)\end{array}$ & $\begin{array}{c}5.17 \\
(.605)\end{array}$ \\
\hline Relação alunos & $\begin{array}{c}5.08 \\
(.576)\end{array}$ & $\begin{array}{c}5.13 \\
(.559)\end{array}$ & $\begin{array}{c}5.08 \\
(.595)\end{array}$ & $\begin{array}{l}5.14^{*} \\
(.547)\end{array}$ & $\begin{array}{c}4.99 \\
(.632)\end{array}$ & $\begin{array}{c}5.17 \\
(.535)\end{array}$ & $\begin{array}{l}5.12^{*} \\
(.572)\end{array}$ \\
\hline Relação colegas & $\begin{array}{c}4.93 \\
(.599)\end{array}$ & $\begin{array}{l}5.01^{* *} \\
(.611)\end{array}$ & $\begin{array}{c}4.99 \\
(.622)\end{array}$ & $\begin{array}{c}5.01 \\
(.606)\end{array}$ & $\begin{array}{c}5.04 \\
(.672)\end{array}$ & $\begin{array}{c}4.98 \\
(.599)\end{array}$ & $\begin{array}{c}5.01 \\
(.601)\end{array}$ \\
\hline Satisfação & $\begin{array}{c}5.35 \\
(.625)\end{array}$ & $\begin{array}{l}5.43^{*} \\
(.569)\end{array}$ & $\begin{array}{c}5.35 \\
(.595)\end{array}$ & $\begin{array}{l}5.43^{* * *} \\
(.583)\end{array}$ & $\begin{array}{c}5.39 \\
(.376)\end{array}$ & $\begin{array}{c}5.37 \\
(.567)\end{array}$ & $\begin{array}{c}5.40 \\
(.588)\end{array}$ \\
\hline $\begin{array}{l}\text { Aceitação de riscos e } \\
\text { iniciativas }\end{array}$ & $\begin{array}{l}4.79 \\
(.615)\end{array}$ & $\begin{array}{l}4.65^{*} \\
(.625)\end{array}$ & $\begin{array}{c}4.68 \\
(.592)\end{array}$ & $\begin{array}{c}4.70 \\
(.643)\end{array}$ & $\begin{array}{c}4.52 \\
(.638)\end{array}$ & $\begin{array}{c}4.65 \\
(.596)\end{array}$ & $\begin{array}{l}4.78^{* *} \\
(.630)\end{array}$ \\
\hline $\begin{array}{l}\text { Autoconceito } \\
\text { profissional docente }\end{array}$ & $\begin{array}{c}5.08 \\
(.441)\end{array}$ & $\begin{array}{c}5.08 \\
(.441)\end{array}$ & $\begin{array}{c}5.06 \\
(.417)\end{array}$ & $\begin{array}{c}5.09 \\
(.453)\end{array}$ & $\begin{array}{c}5.00 \\
(.454)\end{array}$ & $\begin{array}{c}5.08 \\
(.405)\end{array}$ & $\begin{array}{l}5.11^{*} \\
(.459)\end{array}$ \\
\hline
\end{tabular}

Nota: Entre parênteses apresenta-se o valor do desvio-padrão correspondente. * Diferença significativa para $p<.05 ; * *$ diferença significativa para $p<.01 ; * * *$ diferença significativa para $p<.001$ 
Por outro lado, verifica-se a inexistência de efeitos da variável independente orientação religiosa da escola nas restantes dimensões do autoconceito profissional. Relativamente aos anos de serviço, as análises univariadas mostram um efeito de pequena dimensão, na aceitação de riscos e iniciativas, $F(2$, $438)=4.079, p=.018, \eta_{p}^{2}=.011, \pi=.723$. 0 teste post hoc HSD de Tukey, para essas variáveis, mostra uma diferença significativa na aceitação de riscos e iniciativas entre o grupo de professores com 1 a 5 anos de serviço (o grupo de professores menos experiente) e 15 ou mais anos de serviço $(p=.008)$, favorável ao grupo de professores mais experientes.

Por último, há a referir a existência de um efeito de interação entre orientação religiosa da escola e os anos de serviço sobre o autoconceito profissional e as suas dimensões, com exceção da dimensão relação com os colegas. Deste modo, a análise univariada da interação das variáveis orientação religiosa e anos de serviço mostra um efeito estatisticamente significativo, de reduzida dimensão, nas dimensões satisfação, $F(2,438)=6.604, p=.001, \eta_{p}{ }^{2}=.012, \pi=.911$, autoconceito profissional, $F(2,438)=5.614, p=$ $.004, \eta_{p}{ }^{2}=.025, \pi=.858$, competência, $F(2,438)=3.082, p=.047, \eta_{p}^{2}=.014, \pi=.593$ e relação com os alunos, $F(2,438)=3.377, p=.035, \eta_{p}{ }^{2}=.015, \pi=.636$. Os efeitos verificados, resultam principalmente da diferença acentuada nos valores observados no grupo de professores menos experientes ( 1 a 5 anos de tempo de serviço). Assim, verifica-se que a satisfação dos professores de escolas sem orientação religiosa aumenta com o tempo de serviço, enquanto nos professores de escolas com orientação religiosa diminui. No autoconceito profissional (escala total) os valores de autoperceção dos professores de escolas sem orientação religiosa aumentam progressivamente com o tempo de serviço, enquanto os dos professores de escolas com orientação religiosa decrescem, embora de forma pouco pronunciada nos três grupos. Assim, na dimensão competência, os valores de autoperceção aumentam com o tempo de serviço nos professores de escolas sem orientação religiosa, enquanto nos professores de escolas com orientação religiosa se verifica um decréscimo ligeiro. Na dimensão relação com os alunos, verifica-se um aumento nos valores nas autoperceções, à medida que aumentam os anos de serviço nos professores oriundos de escolas sem orientação religiosa, enquanto para os professores de escolas com orientação religiosa os valores de autoperceção decrescem, sendo mais acentuado esse decréscimo no grupo de professores com mais tempo de serviço.

A MANOVA efetuada para analisar os efeitos das três variáveis em estudo sobre a competência percebida do professor, mostra a existência de efeitos principais de pequena dimensão do género, $V=$ $.029, F(4,435)=3.234, p=.012, \eta_{p}{ }^{2}=.029, \pi=.830$ e dos anos de serviço, $V=.050, F(8,878)=2.873, p=$ $.005, \eta_{p}^{2}=.025, \pi=.943$. Verifica-se ainda um efeito de interação entre a orientação religiosa e os anos de serviço, $V=.038, F(4,436)=2.100, p=.033, \eta_{p}^{2}=.019, \pi=.847$. As análises univariadas permitem constatar a existência de efeitos de pequena dimensão do género sobre as competências profissionais e de conduta, $F(1,438)=5.524, p=.019, \eta_{p}{ }^{2}=.012, \pi=.650$ dos anos de serviço sobre a dimensão participação na escola e relação com a comunidade educativa, $F(2,438)=5.301, p=.005, \eta_{p}^{2}=.024$, $\pi=$.836. Os efeitos de interação verificam-se entre a orientação religiosa da escola e os anos de serviço sobre a participação na escola e relação com a comunidade educativa, $F(2,438)=3.878, p=.021, \eta_{p}^{2}=$ $.017, \pi=.700$. A análise post hoc permite constatar a existência de diferenças significativas nos grupos de professores, consoante os anos de serviço considerados, na participação na escola e relação com a comunidade educativa. 0 grupo de professores menos experiente (1 a 5 anos de serviço) apresenta valores significativamente mais baixos do que o grupo experiente (6 a 14 anos de serviço) ( $p=.007)$ e do que o grupo mais experiente (15 ou mais anos de serviço) $(p<.001)$ (Tabela 3).

A análise do efeito de interação das variáveis orientação religiosa da escola e tempo de serviço, para a dimensão participação na escola e relação com a comunidade educativa, mostrou que foi a diferença acentuada observada no grupo de professores com menos tempo de serviço das escolas sem orientação religiosa, a principal responsável por este efeito.

A MANOVA realizada sobre o compósito de variáveis da satisfação de necessidades básicas no trabalho do professor (autonomia e relacionamento), mostra efeitos principais estatisticamente significativos da variável independente género, $V=.020, F(3,436)=2.923, p=.034, \eta_{p}^{2}=.020, \pi=.241$. Todavia, as análises univariadas não revelaram nenhum efeito significativo nas duas dimensões consideradas: autonomia, $F(1.438)=2.747, p=.098$, e relacionamento, $F(1.438)=.429, p=.513$. 
Tabela 3. Médias e desvios-padrão nas dimensões e na escala de competência percebida em função do género, anos de serviço e orientação religiosa da escola

\begin{tabular}{|c|c|c|c|c|c|c|c|}
\hline & \multicolumn{2}{|c|}{ Género } & \multicolumn{2}{|c|}{ Orientação Religiosa } & \multicolumn{3}{|c|}{ Anos de Serviço } \\
\hline & Masculino & Feminino & Não & Sim & $1-5$ & $6-14$ & $\geq 15$ \\
\hline \multirow[t]{2}{*}{ DesEnsApr } & 4.97 & 5.03 & 5.01 & 5.01 & 5.11 & 5.02 & 4.97 \\
\hline & $(.641)$ & $(.492)$ & $(.491)$ & (5.01) & $(.460)$ & $(.571)$ & $(.547)$ \\
\hline \multirow[t]{2}{*}{ CompProfCond } & 5.23 & $5.39 *$ & 5.27 & 5.21 & 5.37 & 5.32 & 5.34 \\
\hline & (.633) & (.449) & $(.563)$ & $(.663)$ & $(.480)$ & $(.558)$ & $(.506)$ \\
\hline \multirow[t]{2}{*}{ PartEscRelComEdu } & 4.49 & 4.51 & 4.48 & 4.51 & 4.10 & 4.51 & $4.62^{* *}$ \\
\hline & $(1.007)$ & $(.950)$ & $(1.011)$ & $(.944)$ & (1.123) & $(.943)$ & $(.899)$ \\
\hline EscaCompPercProf & $\begin{array}{c}4.95 \\
(.606)\end{array}$ & $\begin{array}{c}5.03 \\
(.455)\end{array}$ & $\begin{array}{c}5.00 \\
(.457)\end{array}$ & $\begin{array}{c}5.01 \\
(.457)\end{array}$ & $\begin{array}{c}4.99 \\
(.459)\end{array}$ & $\begin{array}{c}5.01 \\
(.546)\end{array}$ & $\begin{array}{c}5.01 \\
. .498\end{array}$ \\
\hline
\end{tabular}

Nota: Entre parênteses apresenta-se o valor do desvio-padrão correspondente. * Diferença significativa para p <.05; ** diferença significativa para $\mathrm{p}<.01 ; * * *$ diferença significativa para $\mathrm{p}<.001$

DesEnsApr - Desenvolvimento do ensino e aprendizagem; CompProfCond - Competências profissionais e de conduta; PartEscRelComEdu - Participação na escola e relação com a comunidade educativa; EscaCompPercProf - Escala de competência percebida do professor

A ANOVA realizada para a autoestima mostra a inexistência de efeitos significativos do género, $F(1$, $438)=2.782, p=.096$. Constata-se, contudo, um efeito de interação entre o género e os anos de serviço, estatisticamente significativo, de pequena dimensão, $F(2,438)=3.937, p=.020, \eta_{p}^{2}=.018, \pi=.70 .0$ efeito de interação deve-se sobretudo à diferença acentuada entre professoras e professores, no grupo dos professores menos experientes, com os professores a apresentarem níveis de autoestima mais baixos. Por sua vez, não se verificou qualquer tipo de efeito da variável orientação religiosa da escola sobre a autoestima dos professores.

A MANOVA efetuada às diferentes dimensões do compromisso com a escola mostram a existência de efeitos principais do género, de média dimensão, $V=.018, F(4,435)=2.036, p=.045, \eta_{p}^{2}=.18$, $\pi=.658$, e dos anos de serviço, de reduzida dimensão, $V=.039, F(6,874)=2.865, p=.009, \eta_{p}^{2}=.019$, $\pi=.893$, sem que se verifiquem quaisquer efeitos de interação.

As análises univariadas mostram um efeito do género sobre a dimensão compromisso afetivo com a escola, $F(1,438)=5.138, p=.024, \eta_{p}^{2}=.012, \pi=.619$, com as professoras a revelarem um compromisso afetivo com a escola, mais elevado do que os professores (Tabela 4). Revelam, ainda, um efeito significativo da orientação religiosa da escola sobre o compromisso afetivo, $F(1,438)=4.412, p=.029$, $\eta_{p}^{2}=.011, \pi=.591$, com os professores das escolas com orientação religiosa a mostrarem um compromisso afetivo mais elevado do que os professores das escolas sem orientação religiosa (Tabela 4). Ademais, as análises mostram também efeitos tendencialmente significativos da variável anos de serviço sobre o compromisso instrumental, $F(2,438)=5.364, p=.058, \eta_{p}^{2}=.013, \pi=.562$. As análises post hoc, realizadas aos três níveis da variável anos de serviço, evidenciam no compromisso instrumental uma diferença significativa entre o grupo de professores com menos e mais anos de serviço $(p=.008)$. Constata-se, aqui, um padrão decrescente: à medida que os professores avançam no tempo de serviço diminuem os valores médios do compromisso instrumental com a escola (vínculo baseado na necessidade), diferenciando-se de forma significativa os professores com menos e mais tempo de serviço (Tabela 4).

Tabela 4. Médias e desvios-padrão nas dimensões do compromisso com a escola em função do género, anos de serviço e orientação religiosa da escola

\begin{tabular}{|c|c|c|c|c|c|c|c|}
\hline & \multicolumn{2}{|c|}{ Género } & \multicolumn{2}{|c|}{ Orientação Religiosa } & \multicolumn{3}{|c|}{ Anos de Serviço } \\
\hline & Masculino & Feminino & Não & Sim & $1-5$ & $6-14$ & $\geq 15$ \\
\hline Compromisso afetivo & $\begin{array}{c}4.94 \\
(1.083)\end{array}$ & $\begin{array}{c}5.15^{*} \\
(.902)\end{array}$ & $\begin{array}{c}4.94 \\
(1.052)\end{array}$ & $\begin{array}{l}5.16^{*} \\
(.909)\end{array}$ & $\begin{array}{c}4.84 \\
(1.020)\end{array}$ & $\begin{array}{c}5.11 \\
(.966)\end{array}$ & $\begin{array}{l}5.14^{*} \\
(.943)\end{array}$ \\
\hline $\begin{array}{l}\text { Compromisso } \\
\text { normativo }\end{array}$ & $\begin{array}{c}4.00 \\
(1.416)\end{array}$ & $\begin{array}{c}4.16 \\
(1.325)\end{array}$ & $\begin{array}{c}4.16 \\
(1.312)\end{array}$ & $\begin{array}{c}4.08 \\
(1.380)\end{array}$ & $\begin{array}{c}4.34 \\
(1.321)\end{array}$ & $\begin{array}{c}4.16 \\
(1.370)\end{array}$ & $\begin{array}{c}3.99 \\
(1.349)\end{array}$ \\
\hline $\begin{array}{l}\text { Compromisso } \\
\text { instrumental }\end{array}$ & $\begin{array}{c}3.83 \\
(1.169)\end{array}$ & $\begin{array}{c}4.13 \\
(.924)\end{array}$ & $\begin{array}{c}4.13 \\
(.901)\end{array}$ & $\begin{array}{c}4.01 \\
(1.022)\end{array}$ & $\begin{array}{c}4.35 \\
(.930)\end{array}$ & $\begin{array}{c}4.07 \\
(.956)\end{array}$ & $\begin{array}{c}3.95^{*} \\
(1.000)\end{array}$ \\
\hline
\end{tabular}

Nota: Entre parênteses apresenta-se o valor do desvio-padrão correspondente. * Diferença significativa para p<.05; ${ }^{* *}$ diferença significativa para $\mathrm{p}<.01 ;{ }^{* * *}$ diferença significativa para $\mathrm{p}<.001$

Por último, no compromisso com a profissão, a análise univariada não revelou qualquer tipo de efeito introduzido pelo género, orientação religiosa da escola e anos de serviço. 


\section{DISCUSSÃO}

Este trabalho teve como principal objetivo analisar os efeitos do género, anos de serviço e orientação religiosa da escola sobre diferentes componentes da identidade docente. Os resultados apresentados permitiram verificar que o género introduz diferenças na maior parte dos constructos associados à identidade docente, considerados no presente estudo, apesar de os efeitos evidenciados serem, na maior parte dos casos, de reduzida magnitude. Os efeitos das variáveis anos de serviço e orientação religiosa da escola refletiram-se, sobretudo, nas autorepresentações, nomeadamente no autoconceito profissional e na perceção de competência.

No que se refere à orientação ética - orientação para o cuidado e orientação para a justiça, os resultados mostraram que esta variável é apenas diferenciada em função do género, com as professoras a manifestarem uma atitude global mais favorável na orientação ética. Estes resultados suportam, em parte, o posicionamento dos autores que defendem a possibilidade de homens e mulheres poderem deliberar com base em princípios da ética do cuidado e da justiça, mas não apoiam a afirmação de que haveria por parte das mulheres o predomínio de deliberações orientadas pela ética do cuidado (e.g., Gilligan, 1982; Gilligan \& Attanucci, 1988; Jaffe \& Hyde, 2000; Karniol et al., 2003). Do mesmo modo, os resultados não sustentam a tese das autoras que defendem que os indivíduos do género masculino deliberariam fundamentalmente a partir de critérios de justiça (Gilligan, 1982; Gilligan \& Attanucci, 1988). Adicionalmente, os resultados contrariam as perspetivas dos autores que defendem a inexistência de diferenças significativas na orientação ética em função do género (e.g., Vikan et al., 2005).

Portanto, este estudo evidencia a possibilidade de identificar domínios na identidade docente que envolvem processos deliberativos baseados em critérios/princípios de justiça e cuidado, independentemente do género. A cultura do cuidado não é exclusiva dos professores do género feminino, assim como a cultura da justiça não o é dos professores do género masculino. Reforça-se, assim, a ideia de que a maturidade ética dos professores se manifesta na habilidade de manter a perspetiva do cuidado e da justiça de forma equilibrada e complementar nas práticas dos professores. Uma análise crítica e reflexiva dos professores, sobre esta matéria, pode contribuir de forma decisiva para o reforço da identidade profissional dos professores e ter repercussões numa prática profissional intencionalmente ética. Adicionalmente, é crucial não perpetuar o discurso da feminilidade e diferença de gênero principalmente quando se busca recuperar o cuidado como uma atividade de valor e necessidade. Há que reconhecer o cuidado como uma dimensão importante da identidade dos professores, e não apenas como uma atividade exclusivamente maternal ou parental.

Quanto às diferenças no autoconceito profissional docente e suas dimensões, os resultados mostraram que os professores do género feminino apresentam nas dimensões relação com os colegas e na dimensão satisfação profissional do autoconceito profissional valores significativamente mais elevados, enquanto os professores do género masculino apresentam valores superiores na dimensão aceitação de riscos e iniciativas. Os resultados encontrados estão em desacordo com os do estudo de Gonçalves e Veiga (2006), coincidindo apenas no facto de não terem sido encontradas diferenças na dimensão relação com os alunos. Por sua vez, os professores que lecionam em escolas com orientação religiosa autopercecionaram-se de forma mais favorável nas dimensões relação com os alunos e satisfação relativamente aos seus congéneres de escolas sem orientação religiosa. A diferença verificada parece apontar para diferenças devidas a aspetos de natureza contextual, em linha com o pensamento dos autores que enfatizam a influência de fatores externos e ambientais no desenvolvimento da identidade e em particular da identidade profissional docente (e.g., Dubar, 1997; Bronfenbrenner, 1996; Flores \& Day, 2006). Estes resultados necessitam, contudo, de um estudo aprofundado em futuras investigações, que considere efeitos de outras variáveis no desenvolvimento da identidade docente, como sejam a incorporação da missão e dos valores da escola pelos professores e os principais indicadores do clima de escola (Whitaker et al., 2010). Por último, os resultados mostram que o autoconceito profissional aumenta com a experiência na dimensão aceitação de riscos e iniciativas, ou seja, os professores menos experientes consideram ter uma menor propensão para aceitar riscos e tomar iniciativas nas suas práticas em relação aos professores experientes. Estes resultados estão em consonância com os de Veiga et al. (2003), à exceção das diferenças por eles encontradas na dimensão satisfação e na dimensão relação alunos. Contudo, estão em desacordo com os revelados pelo estudo de Gonçalves e Veiga (2006), que não encontraram quaisquer tipos de diferenças provocadas pelo efeito da variável anos de serviço no autoconceito profissional e respetivas dimensões. Contrariam, também, os resultados do estudo de Fonseca e Veiga (2010), quando referem que o autoconceito profissional se torna mais «negativo» à medida que aumenta o tempo de serviço dos professores. Estas discrepâncias nos resultados poderão ter a ver com os critérios utilizados na seleção dos professores participantes, oriundos de contextos organizacionais diferentes, uns do ensino particular e cooperativo e outros do ensino público, ao que acresce o facto de o estudo de Fonseca e Veiga (2010) contemplar apenas os professores dos 
departamentos disciplinares de português e de matemática do 2.ํo ciclo. Daí a necessidade de confirmar estes resultados com um número de participantes mais significativo e de diferentes contextos, superando, assim, possíveis limitações deste estudo neste domínio.

A análise dos efeitos de interação das variáveis orientação religiosa e tempo de serviço para as dimensões satisfação, autoconceito profissional, competência e relação alunos, mostrou que a principal responsável pelas diferenças verificadas foi a diferença substancial verificada no grupo de professores com menos tempo de serviço.

Este é um resultado importante, e uma das implicações relevantes deste estudo a ter em conta pelas lideranças escolares, na medida em poderão estar em causa aspetos que remetem para a forma como se processa o acolhimento e acompanhamento dos professores em início de carreira permitindolhes justificar a implementação de ações de melhoria para o desenvolvimento da identidade profissional desses professores.

Como vimos, os resultados na competência profissional percebida do professor mostraram que os professores do género feminino se autopercecionam como mais competentes na dimensão competências profissionais e de conduta em relação aos professores do género masculino. Este resultado está em desacordo com os encontrados por outros autores, segundo os quais os professores do género masculino expressavam níveis de perceção de competência percebida mais elevados do que os professores do género feminino (e.g., Antoniou, Polychroni, \& Vlachakis, 2006; Chaplain, 1995; Gonçalves \& Veiga, 2006; Klasser \& Chiu, 2010). No entanto, vão no mesmo sentido dos resultados do estudo de Ruivo et al. (2008), no qual participaram 3252 professores portugueses do ensino público. 0 argumento utilizado por Mowday et al., 1979) pode, em parte, justificar estes resultados, quando referem que as mulheres têm lutado muito para obter estatutos que exigem maiores níveis de competência percebida e desempenhos profissionais eficazes.

Por sua vez, os professores menos experientes (1 a 5 anos de serviço) consideram-se menos competentes no que respeita à participação na gestão da escola e no relacionamento com a comunidade educativa comparativamente aos professores experientes ( 6 a 14 anos de serviço) e muito experientes (15 ou mais anos de serviço). Estes resultados estão em consonância com os resultados dos estudos de Wolters e Daugherty (2007) e Canrinus et al. (2012), que apontam para efeitos modestos e/ou inexistentes da experiência sobre a autoeficácia. Os resultados encontrados na dimensão participação na escola e relação com a comunidade educativa estão, ainda, em conformidade com os resultados dos estudos de Tschannen-Moran e Hoy (2007), Wolters e Daugherty (2007), segundo os quais os professores apresentam níveis fracos na autoeficácia nos primeiros anos de ensino.

Outra das implicações interessantes deste estudo resulta da análise do efeito de interação das variáveis orientação religiosa e tempo de serviço para a dimensão participação na escola e relação com a comunidade educativa, mostrando, mais uma vez, que foi a diferença acentuada observada no grupo de professores com menos tempo de serviço, a principal responsável pela diferença verificada, o que reforça a ideia da importância do suporte institucional para a construção de uma identidade docente sólida e positiva.

No que concerne à satisfação de necessidades de autonomia e relacionamentos, os professores do género feminino e masculino apresentam autoperceções muito semelhantes. Também os anos de serviço não introduzem qualquer variabilidade nos fatores que compõem a satisfação de necessidades psicológicas básicas no trabalho dos professores, o que reforça os resultados encontrados por Wolters e Daugherty (2007) e Canrinus et al. (2012) nos seus estudos, que enfatizam o facto de a experiência profissional não introduzir mudança nos níveis motivacionais dos professores (autonomia, autoeficácia, relacionamento e satisfação no trabalho).

Relativamente à autoestima dos professores participantes, os resultados mostram a inexistência de diferenças significativas devidas ao género, em sintonia com os resultados de estudos de investigadores nacionais e internacionais (e.g., Peixoto, 2003; Veiga, 1995; Wylie, 1979). Verificámos, ainda, a inexistência de diferenças introduzidas quer pela orientação religiosa da escola quer pelos anos de serviço na autoestima dos professores, um contributo teórico importante deste estudo, que carece de confirmação. Os resultados aqui apresentados estão em linha com os encontrados por vários autores que salientam a inexistência de diferenças na autoestima introduzidas pela experiência (e.g., Veiga, 1995; Wylie, 1979). Todavia, enquanto nos professores do género masculino os valores da autoestima aumentam com os anos de serviço, nos professores do género feminino os valores da autoestima tendem a baixar à medida que avançam na carreira profissional, devido fundamentalmente à diferença de valores verificados no grupo dos professores com menos tempo de serviço, que é desfavorável para os professores de género masculino.

Este estudo parece evidenciar a alta estabilidade da autoestima dos professores. De acordo com Hart, Atkins e Tursi (2006), a alta estabilidade da autoestima e o facto de os indivíduos poderem ser 
amplamente tendenciosos na forma de avaliar o mundo, de forma negativa ou positiva, pode contribuir para esse facto, o que é relativamente novo e rico para a compreensão do desenvolvimento da autoestima.

Nos resultados obtidos no compromisso do professor com a escola, verificaram-se diferenças, sobretudo, no compromisso afetivo e no compromisso instrumental. Na dimensão compromisso afetivo, os professores do género feminino percecionam-se como mais comprometidos com a escola do que os seus congéneres do género masculino. Este resultado corrobora os de outros estudos nos quais as mulheres apresentam um compromisso significativamente superior com a organização, comparativamente aos homens (e.g., Mowday et al., 1979). Na mesma linha, os professores que lecionam em escolas com orientação religiosa revelam-se mais comprometidos no plano afetivo com a escola do que os professores que lecionam em escolas sem orientação religiosa. Este é mais um resultado importante deste estudo, que carece de confirmação em novos estudos, com um número de participantes de escolas com e sem orientação religiosa mais adequado, que permitam ultrapassar possíveis limitações desta investigação e identificar a razão ou razões que justificam a diferença verificada no fator compromisso afetivo com escola, que favorece os professores das escolas com orientação religiosa.

Por sua vez, os resultados apresentados mostram também que à medida que aumenta o tempo de serviço aumenta o compromisso afetivo dos professores com a escola, com uma diferença significativa entre o grupo dos professores com menos e mais anos de tempo de serviço. Na dimensão compromisso instrumental, à medida que aumentam os anos de serviço dos professores diminui o compromisso instrumental, com os professores menos experientes a revelarem níveis de compromisso instrumental mais elevados relativamente aos professores muito experientes. Como consequência disto pode pensar-se em, do ponto de vista organizacional, usar os professores com mais tempo de serviço para ajudar a integrar os mais novos.

Adicionalmente, estes resultados são um contributo teórico importante para o estudo do compromisso do professor com a escola ao evidenciarem a complementaridade entre o compromisso normativo, o compromisso afetivo e instrumental, vistos como componentes e não como tipos de compromisso, atendendo a que a relação de um funcionário com uma organização pode refletir diferentes graus de todas essas três componentes, à semelhança do verificado noutros contextos organizacionais e em linha com o modelo tridimensional do compromisso organizacional (Allen \& Meyer 1996; Meyer \& Allen, 1991).

$\mathrm{Na}$ dimensão compromisso normativo com a escola, constata-se a inexistência de diferenças significativas introduzidas pelas variáveis independentes. Os resultados apresentados relativamente ao compromisso do professor com a escola, estão na generalidade em dissonância com os encontrados por Wolters e Daugherty (2007) e Canrinus et al. (2012), à exceção na dimensão compromisso normativo com a escola, que apontam para a inexistência de efeitos da experiência docente sobre o compromisso dos professores. Para explicar estes resultados poderão contribuir um conjunto de razões de natureza individual e organizacional, nomeadamente as características específicas dos professores participantes, o tipo de formação inicial e contínua instituída, o papel das lideranças intermédias e de topo na motivação dos professores, o clima de escola e a especificidade das condições sociopolíticas de trabalho.

Por último, é de salientar que a inexistência de efeitos introduzidos pelo género e os anos de serviço no compromisso com a profissão corroboram os resultados obtidos nos estudos de Canrinus et al. (2012) e Skaalvik e Skaalvik (2011). Contudo, estes resultados carecem de uma discussão e estudos mais abrangentes, pois eventuais efeitos positivos ou negativos no compromisso com a profissão podem deverse a fatores não contemplados neste estudo, nomeadamente o clima de escola, o suporte proporcionado aos professores pelas lideranças, em especial aos professores em início de carreira e a fatores de natureza sociopolítica, geralmente associados à insatisfação docente. Abre-se, aqui, uma janela de oportunidade para a implementação de novos estudos que possam melhor entender o compromisso do professor com a profissão.

Por fim, os resultados observados no presente estudo levantam questões que deverão ser esclarecidas em futuros trabalhos no sentido de aprofundar os resultados aqui apresentados, com recurso a professores participantes de outros contextos escolares e a instrumentos de recolha de dados diversificados e que contemplem outras variáveis antes sugeridas, relacionadas com o desenvolvimento da identidade docente. Será seguramente um contributo importante para o estudo e desenvolvimento da identidade dos professores e, consequentemente, para a compreensão e valorização dos professores, condição sine qua non para uma educação de qualidade para todos. 


\section{REFERÊNCIAS}

Allen, N., \& Meyer, J. (1996). Affective, continuance, and normative commitment to the organization: An examination of construct validity. Journal of Vocational Behavior, 49, 252-276. http://dx.doi.org/10.1006/jvbe.1996.0043

Antoniou, A., Polychroni, F., \& Vlachakis, A. (2006). Gender and age differences in occupational stress and professional burnout between primary and high-school teachers in Greece. Journal of Managerial Psychology, 21, 682-69. http://dx.doi.org/10.1108/02683940610690213

Aydin, A., Sarier, Y., \& Uysal, S. (2011). The effect of gender on organizational commitment of teachers: A meta analytic analysis. Educational Sciences: Theory \& Practice, 11 (2), 628-632.

Aznar, M., Fernández, I., Quevedo, R., \& Abella, M. (2003). Diferencias en autoestima en función del género. Análise y Modificación de Conducta, 29 (123), 51-78.

Balay, R. (2000). Organizational commitment of administrators and teachers in private and public secondary schools: Ankara sample. Unpublished doctoral dissertation, Ankara University, Ankara, Turkey.

Banks, S., \& Nohr, K. (2003). Teaching practical ethics for the social professions. Copenhagen: Feset.

Bandura, A. (1977). Social learning theory. Englewood Cliffs, N.J.: Prentice-Hall.

Beijaard, D., Meijer, P. C., \& Verloop, N. (2004). Reconsidering research on teachers professional identity. Teaching and Teacher Education, 20, 107-128. http://dx.doi.org/10.1016/j.tate.2003.07.001

Benhabib, S. (1992). Una revision del debate sobre las mujeres y la teoria moral. ISEGORÍA, 6, 37-63. http://dx.doi.org/10.3989/isegoria.1992.i6.323

Bong, M., \& Skaalvik, E. M. (2003). Academic self-concept and self-efficacy: How different are they really? Educational Psychology Review, 15, 1-40. http://dx.doi.org/10.1023/A:1021302408382

Bronfenbrenner, U. (1996). A ecologia do desenvolvimento humano. Porto Alegre: Artes Médicas.

Canrinus, E., Helms-Lorenz, M., Beijaard, D., Buitink, J., \& Hofman, A. (2012). Self-efficacy, job satisfaction, motivation and commitment: exploring the relationships between indicators of teacher's professional identity. European Journal of Psychology of Education, 27, 115-132. http://dx.doi.org/10.1007/s10212-011-0069-2

Chaplain, R. (1995). Stress and job satisfaction: A study of English primary school teachers. Educational Psychology, 15, 473-489. http://dx.doi.org/10.1080/0144341950150409

Cohen, J. (1992). A power primer. Psychological Bulletin, 112, 155-159. http://dx.doi.org/10.1037/00332909.112.1.155

Day, C., \& Gu, Q. (2007). Variations in the conditions for teachers' professional learning and development: Sustaining commitment and effectiveness over a career. Oxford Review of Education, 33, 423-443. http://dx.doi.org/10.1080/03054980701450746

Deci, E., \& Ryan, R. (2000). The "What" and "Why" of goal pursuits: Human needs and self-determination of behavior. Psychological Inquiry, 11, 227-268. http://dx.doi.org/10.1207/S15327965PLI1104_01

Dubar, C. (1997). A socialização - Construção das identidades sociais e profissionais. Porto: Porto Editora.

Erikson, E. (1982). The life cycle completed. New York: W.W. Norton.

Flores, M., \& Day, C. (2006). Contexts which shape and reshape new teachers' identities: A multiperspective study. Teaching and Teacher Education, 22, 219-232. http://dx.doi.org/10.1016/j.tate.2005.09.002

Fonseca, S., \& Veiga, F. (2010). Autoconceito profissional dos professores de ciências e matemática do $2^{\circ}$ ciclo do ensino básico: Adequação e relação com o tempo de serviço, a formação e a cidadania. In Actas do VII Simpósio Nacional de Investigação em Psicologia, Braga.

Gilligan, C. (1982). In a different voice: Psychological theory and woman's development. Cambridge: Harvard University Press.

Gilligan, C., \& Attanucci, J. (1988). Two moral orientations: Gender differences and similarities. MerrillPalmer Quarterly, 34 (3), 223-237.

Gonçalves, J. (2000). A carreira das professoras do ensino primário. In A. Nóvoa (Org.). Vidas de Professores (pp. 141-169). Porto: Porto Editora.

Gonçalves, V. F., \& Veiga, F. H. (2006). Autoconceito profissional dos professores. In Investigação em Psicologia, Actas VI Simpósio Nacional de Investigação em Psicologia. Évora: Universidade de Évora

Granjo, M. (2015). Contributo para o estudo a identidade docente: Autoperceções dos professores do ensino particular e cooperativo, o papel da ética e a mediação da autoestima e dos relacionamentos no compromisso afetivo do professor com a escola e a profissão (Tese de Doutoramento não publicada). ISPA-Instituto Universitário de Ciências Psicológicas, Sociais e da Vida, Lisboa.

Gursel, M., Sunbul, A., \& Sari, H. (2002). An analysis of burnout and job satisfaction between Turkish headteachers and teachers. European Journal of Psychology of Education, 17, 35-45. http://dx.doi.org/10.1007/BF03173203 
Hart, D., Atkins, R., \& Tursi, N (2006). Origins and developmental influences on self-esteem. In M. Kernis (Ed.), Self-esteem issues and answers. A sourcebook of current perspectives, (pp.157-162). New York: Psychology Press.

Harter, S. (1999). The construction of the self: A developmental perspective. New York: Guilford Press.

Hattie, J. (1992). Self-concept. New Jersey: Lawrence Erlbaum Associate.

Huberman, M. (2000). O ciclo de vida profissional dos professores. In A. Nóvoa (Org.). Vidas de professores, (pp. 31-61). Porto: Porto Editora.

Ilardi, B., Leone, D., Kasser, R., \& Ryan, R. (1993). Employee and supervisor ratings of motivation: Main effects and discrepancies associated with job satisfaction and adjustment in a factory setting. Journal of Applied Social Psychology, 23, 1789-1805. http://dx.doi.org/10.1111/j.15591816.1993.tb01066.x

Jaffe, S., \& Hyde, J. (2000). Gender differences in moral orientation: A meta-analysis. Psychological Bulletin, 126, 703-726. http://dx.doi.org/10.1037/0033-2909.126.5.703

Karniol, R., Grosz, E., \& Schorr, I. (2003). Caring, gender role orientation, and volunteering. Sex roles, 49, 11-19. https://doi.org/10.1023/A:1023953401662

Kelchtermans, G. (2005). Teachers' emotions in educational reforms: Self-understanding, vulnerable commitment and micropolitical literacy. Teaching and Teacher Education, 21, 995-1006. http://dx.doi.org/10.1016/j.tate.2005.06.009

Klasser, R., \& Chiu, M. (2010). Effects on teachers' self-efficacy and job satisfaction: Teacher gender, years of experience, and job stress. Journal of Educational Psychology, 3, 741-756. http://dx.doi.org/10.1037/a0019237

Kohlberg, L. (1984). Psychology of moral development: The nature and validity of moral stages, in Essays on Moral Development, Vol. 2. San Francisco: Harper and Row.

Korthagen, F. (2004). In search of the essence of a good teacher: Towards a more holistic approach in teacher education. Teaching and Teacher Education, 20, 77-97. http://dx.doi.org/10.1016/j.tate.2003.10.002

Kooij, D., de Lange, A., Jansen, P., \& Dikkers, J. (2008). Older workers' motivation to continue to work: Five meanings of age. Journal of Managerial Psychology, 23, $364 \quad-\quad 394$. http://dx.doi.org/10.1108/02683940810869015

Marôco, J. (2011). Análise estatística com o SPSS statistics. Lisboa: Edições Silabo Lda.

Markus, H., \& Wurf, E. (1987). The dynamic self-concept: A social psychological perspective. Annual Review of Psychology, 38, 299-337. http://dx.doi.org/10.1146/annurev.ps.38.020187.001503

Marsh, H., \& Ayotte, V. (2003). Do multiple dimensions of self-concept become more differentiated with age? The differential distinctiveness hypothesis. Journal of Educational Psychology, 95, 687-706. http://dx.doi.org/10.1037/0022-0663.95.4.687

Marsh, H., \& Roche, L. (2000). Multiple dimensions of university teacher self-concept: Construct validation and the influence of students evaluations of teaching. Instructional Science, 28 (5), 439468.

Messer, B., \& Harter, S. (1986). Manual for the adult self-perception profile. University of Denver.

Meyer, J., \& Allen, N. (1991). A three-component conceptualization of organizational commitment. Human Resources Management Review, 1, 61-89. http://dx.doi.org/10.1016/1053-4822(91)90011-Z

Mowday, R., Steers, R., \& Porter, L. (1979). The measurement of organizational commitment. Journal of Vocational Behavior, 14, 224-247. http://dx.doi.org/10.1016/0001-8791(79)90072-1

Moog, F. (2012). A quoi sert l'école catholique - Sa mission d'évangélisation dans la societé actuelle. Ed. Bayard.

Nascimento, J., Lopes, A., \& Salgueiro, M. (2008). Estudo sobre a validação do "Modelo de Comportamento Organizacional" de Meyer e Allen para o contexto português. Comportamento Organizacional e Gestão, 14 (1), 115-133.

Noddings, N. (1999). Introduction. In M. Katz, N. Noddings, \& K. Stike, K. (Eds) Justice and caring. The search for common ground in education, (pp. 1-4). New York: Teachers college press.

Papa Francisco (2014, 13 fev.). Discorso del Santo Padre Francesco ai partecipanti alla plenária della congragazione per léducazione cattolica (Degli Istituti di Studi). Libreria Editrice Vaticana.

Pedro, N., \& Peixoto, F. (2006). Satisfação profissional e autoestima em professores do $2^{\text {o }}$ e $3^{\circ}$ ciclos do ensino básico. Análise Psicológica, 24, 247-262. https://doi.org/10.14417/ap.167

Peixoto, F. (2003). Autoestima, autoconceito e dinâmicas relacionais em contexto escolar (Tese de Doutoramento não publicada). Universidade do Minho, Braga.

Perrenoud, P. (2000). 10 novas competências para ensinar. Porto Alegre: Artmed Editora. 
Razak, N., Darmawan, \& Keeves, J. (2009). Teacher commitment. In L. J. Saha \& A. G. Dworkin (eds.). International Handbook of Research on Teachers and Teaching (pp. 343-360). Norwell, MA: Springer.

Rawls, J. (1971/1993). Uma teoria da justiça. Lisboa: Editorial Presença.

Rego, A., Cunha, M., \& Souto, S. (2007). Espiritualidade nas organizações e comprometimento organizacional. RAE - Eletrónica, 6, 1-27. http://dx.doi.org/10.1590/S1676-56482007000200003

Ruivo, J., Sebastião, J., Rafael, J., Afonso, P., \& Nunes, S. (2008). Ser professor - Satisfação profissional e papel das organizações de docentes (Um estudo nacional). Castelo Branco: Instituto Politécnico de Castelo Branco. Associação Nacional de Professores.

Sachs, J. (2005). Teacher education and the development of professional identity. Learning to be a teacher. In P. Denicolo \& M. Kompf (Eds.), Connecting policy and practice: Challenges for teaching and learning in schools and universities, (pp. 5-21). Oxford: Routledge.

Skaalvik, E. \& Skaalvik, S. (2011). Teacher job satisfaction and motivation to leave the teaching profession: Relations with school context, feeling of belonging, and emotional exhaustion. Teaching and Teacher Education, 27, 1029-1038. http://dx.doi.org/10.1016/j.tate.2011.04.001

Tabachnick, B., \& Fidell, L. (2012). Using multivariate statistics (6a Edição). New York, NY: HarperCollins College Publishers.

Tabassum, F., \& Ali, M. (2012). Professional self-esteem of secondary school teachers. Asian Social Science, 8, 206-21. http://dx.doi.org/10.5539/ass.v8n2p206

Tschannen-Moran, M., \& Hoy, A. W. (2007). The differential antecedents of self-efficacy beliefs of novice and experienced teachers. Teaching and Teacher Education, 23, 944-956. http://dx.doi.org/10.1016/j.tate.2006.05.003

Tschannen-Moran, M., Hoy, A. W., \& Hoy, W. (1998). Teacher efficacy: Its meaning and measure. Review of Educational Research, 68, 202-248. http://dx.doi.org/10.3102/00346543068002202

Veiga, F. (1995). Transgressão e autoconceito dos jovens na escola. Lisboa: Fim de Século

Veiga, F., Roque, P., Antunes, J., Fernandes, L., \& Guerra, T. (2003). Autoconceito profissional dos professores: Construção de uma escala de avaliação. In Actas do VII Congresso Galaico-português de Psicopedagogia. Corunha: Universidade da Corunha.

Veiga, F., Gonçalves, V., Caldeira, M., \& Roque, P. (2006). Representações dos professores acerca de simesmos: Adaptação portuguesa da escala "Teacher self-concept evaluation scale". Estudo apresentado no XIV Colóquio Internacional da AFIRSE/AIPELF, realizado em 16, 17 e 18 de Fevereiro de 2006, na Faculdade de Psicologia e de Ciências da Educação da Universidade de Lisboa.

Verhach, E. (2014). 0 futuro da formação cristã e do acompanhamento espiritual dos docentes católicos nas escolas. Revista de Educação e Catequese, 30, 93-99.

Vikan, A., Camino, C., \& Biaggio, A. (2005). Note on a cross-cultural test of Gilligan's ethic of care. Journal of Moral Education, 34, 107-111. https://doi.org/10.1080/03057240500051105

Villa, A., \& Calvete E. (2001). Development of the teacher self-concept. Evaluation scale and its relation to burnout. Studies in Educational Evaluation, 27, 239-255. http://dx.doi.org/10.1016/S0191491X(01)00028-1

Whitaker, T., Whitaker, B., \& Lumpa, D. (2009). Motivating and inspiring teachers: The educational leader's guide for building staff morale (2nd Edition). Nova Iorque: Eye on Education.

Wolters, C., \& Daugherty, S. (2007). Goal structures and teachers' sense of efficacy: Their relation and association to teaching experience and academic level. Journal of Educational Psychology, 99, 181193. http://dx.doi.org/10.1037/0022-0663.99.1.181

Wylie, R. (1979). The self-concept (vol. 2): Theory and research on selected topics. Lincoln: University of Nebraska Press.

Historial do artigo

Recebido $\quad 07 / 10 / 2017$

Aceite 18/11/2018

Publicado 12/2018 